\title{
The Year Gone By: SFM Academic Activities 2017
}

\author{
Ashok Khurana ${ }^{1} \cdot$ Ratna D. Puri ${ }^{2}$
}

Received: 3 April 2018/Accepted: 4 April 2018/Published online: 10 April 2018

(C) Society of Fetal Medicine 2018

2017 saw the Society of Fetal Medicine (SFM) steadily pursuing its mission of opening minds to fetal needs using a combination of small steps and rapid strides to work towards fulfilling its vision of offering every fetus an optimal outcome. This global forum of Indian origin continues to be an evolving multidisciplinary organization with members from the fields of obstetrics, radiology and imaging, genetics, fetal medicine, pediatrics, pediatric surgery, neonatology, pediatric cardiology, cardiac surgery, laboratory medicine, laboratory technology, infertility, community medicine, medical statistics, medical ethics, social work and psychology. The Society's primary objectives are to promote and expand education and research in fetal medicine and facilitate rapid translation of research findings into clinical application. The society continues to encourage and organize professional education programs, public awareness forums and is currently actively enunciating guidelines for standards of practice. With over 1500 members, delegates at our educational programs span across all continents.

Academic activities included an appropriately variable skill level, size and content to meet the needs of different geographical regions and a heterogeneous delegate and member profile.

The annual fetal cardiology congress of SFM, Fetal CardioCon, was held in New Delhi in April and saw unprecedented delegate strength. The feedback was

Ashok Khurana

ashokkhurana@ashokkhurana.com

1 The Ultrasound Lab, C584 Defence Colony, New Delhi 110024, India

2 Institute of Medical Genetics and Genomics, Sir Ganga Ram Hospital, Rajinder Nagar, New Delhi 110060, India reassuring and the entire exercise went a long way to fulfill the needs of this emerging segment of fetal and neonatal cardiac healthcare. Rabih Chaoui and Fred Ushakov were on the overseas faculty and were greatly appreciated. The Indian faculty was a blend of youth and wisdom, and the quality of proceedings was rated as phenomenal by the delegates. The presentations were a visual feast and resulted in a large number of national and international publications. This showcase of high quality ultrasound and clinical work went a long way to educate all segments: the newly initiated, the practitioner and the superspecialist.

FetalMed, our biennale, was held in Gurgaon in September and saw a large international faculty give presentations and interact on the themes of fetal growth, hypertensive disorders, current and emerging fetal genetics scenario, basic and advanced fetal ultrasound, fetal MRI and community fetal medicine practice. The meeting hosted the 13th Asia Pacific Congress of Maternal-Fetal Medicine and the combination went a long way in understanding the similarity of skills and training programs needed in various parts of the Asia-Pacific region. Live workshops on protocol based evaluation and focused groups in clinical genetics were organized on the first day and saw immense interaction between faculty and delegates. The meeting saw the submission of multiple abstracts from young delegates, noting the quality of work being performed in multiple regions of the country. Outstanding abstracts were selected for oral presentations during the various sessions that were monitored and discussed by national and international experts. These incentives have encouraged young talent to write and publish their work in peer-reviewed journals. Radical changes in the approach to fetal genetics were discussed threadbare and an SFM position statement is in the offing. The foreign faculty included Aris Papageorghiou, George Yeo, Howard 
Cuckle, TY Leung, Liona Poon, Yves Ville, Katia Bilardo, Laurent Salomon, Jon Hyett, Simon Meagher, Richard Choy, Chung Ming Chor, Kim Sun Khaw, Lai Wa Law, Wai Lam Lau, Shashikant Kulkarni, Mark I Evans, Lay Kok Tan, Muniswaran Ganeshan, Sabaratnam Arulkumaran, Torbjorn Eggebo, Andrew Shennan and Tim Draycott. Delegates from 27 countries attended the congress.

The official journal of the society, "Journal of Fetal Medicine" regularly brings forth four issues in a year. Year 2017 has seen important contributions to the practice of fetal medicine in India including the implications of the ACMG non-invasive guidelines for the Indian patient, prenatal screening strategies in regions with limited resources and the experience in India, placental pathology and stillbirth, strategies for fetal therapy etc. and multiple brief communications of salient fetal findings.

A first of its kind, live and hands on workshop and course on basic interventional procedures in fetal medicine was conducted in New Delhi in May. The impeccable organizational abilities of the hosting team, the department of Obstetrics and Gynecology of Maulana Azad Medical, were clearly in evidence. Excellent live diagnostic procedure demonstrations and the hands on simulators helped the delegates hone their skills. This was a certificate course and has proved very useful in taking simple techniques to small cities. Animated discussions marked the event and put together clinical fetal medicine, imaging, genetics and laboratory technology on the same page.

SFM regional chapters and spirited teams in Ludhiana, Patiala, Gurgaon, Kochi, Hyderabad, New Delhi, Kolkata, Ahmedabad and Rajkot organized themed annual and quarterly meetings. Themes centered on everyday scenarios in fetal diagnosis and management and received excellent feedback. SFM encourages such regional activities and logistical guidelines are in place.

SFM also set out to mark its contribution and presence in regional specialty meetings organized by like-minded societies and organizations. Such academic collaboration was evident in the ultrasound meet in Jodhpur and the fetal medicine meet in Kochi. Jodhpur focused on enhancing basic skills to an advanced level using a combination of live workshops, didactic lectures and panel discussions. The Kochi meet saw the presence of Basky Thilaganathan, editor of the extremely prestigious "White Journal", Ultrasound in Obstetrics and Gynecology. The presentations were incisive and a definite eye opener to changing concepts in basic and advanced maternal and fetal healthcare. SFM looks forward to such local and national cobranding with the aim of fulfilling its mission.

The year-end saw two more member-centric great achievements. The first was the launch of our mobile-based SFM app with the first calculator. This has been an instant success. Future plans include more calculators, readily accessible scientific content and instant access to a payment gateway. The other achievement was obtaining a substantially discounted registration fee for SFM members at the World Congress of the International Society of Ultrasound in Obstetrics and Gynecology (ISUOG) that will be held in Singapore in October 2018. Members are encouraged to register early and send abstracts as well. The academic association of SFM with ISUOG continues and our major events are all ISUOG Approved Courses.

We look forward to a fruitful 2018. 